\title{
Polymethoxylated Flavone Content of Major Cultivars and Local Accessions of Citrus Cultivated in Kagoshima, Japan
}

\author{
Masashi Yamamoto $^{1 *}$, Natsuki Nishiguchi ${ }^{1}$, Atsushi Shimada ${ }^{1}$ and Ryoji Matsumoto ${ }^{2 * *}$ \\ ${ }^{1}$ Faculty of Agriculture, Kagoshima University, Korimoto, Kagoshima 890-0065, Japan \\ ${ }^{2}$ Faculty of Agriculture, Saga University, Honjo-cho, Saga 840-8502, Japan
}

In this study, we cultivated 32 major citrus cultivars and local accessions of Kagoshima and one control accession to elucidate the polymethoxylated flavone contents with the purpose of providing information for citrus consumers to promote citrus production in the region. All trees were grafted on trifoliate orange rootstock and cultivated in Kagoshima City, Japan. In general, the Brix values and fruit weights of the major cultivars were higher and heavier than those of the local accessions. The ascorbic acid content of Tankan and Rokugatsu Mikan was high and that of Shiikuwasha, Kabuchii, and Shiikuu was very low. For analysis of polymethoxylated flavones (PMF), important health-promoting components sinensetin, nobiletin, heptamethoxyflavone, and tangeretin were quantified. Cultivar and accession differences in the PMF content were also observed. Nobiletin and tangeretin were found to be major PMF in most accessions, and heptamethoxyflavone was not detected in some species such as Kishu Mikan, Ponkan, Shiikuwasha, and Kuroshima Mikan. In the major cultivars, the PMF contents of Kishu Mikan, Ponkan, and Tankan were found to be high. In the local citrus accessions, Shiikuwasha, its relative, and Kishu Mikan were found to have the highest PMF content, and those of Kabuchii and Kuroshima Mikan were also high. Major cultivars Satsuma mandarin, pummelo, and its relative and the local accession Kunenbo contained little PMF.

Key Words: Amami Islands, ascorbic acid, nobiletin, ponkan, tankan.

\section{Introduction}

Citrus fruit is a rich source of phytonutrients. Among these, polymethoxylated flavones (PMF) are an important health-promoting component. They show anticancer, anti-tumor, and anti-hyperglycemic effects (Kawai et al., 1999; Lee et al., 2010; Minagawa et al., 2001; Miyata et al., 2008), as well as effects against Alzheimer's disease (Nady et al., 2017; Onozuka et al., 2008). A previous study (Nogata et al., 2006) reported that large accession differences exist in the PMF content. In general, the PMF content is high in mandarin, but low in citron and pummelo. However, the contents of not all mandarins were high. Thus, elucidation of the PMF content of each citrus accession is essential.

Kagoshima Prefecture is located in the southern part of Japan. Its climate is temperate to subtropical. Since

Received; November 20, 2018. Accepted; December 28, 2018.

First Published Online in J-STAGE on March 2, 2019.

No conflicts of interest declared.

* Corresponding author (E-mail: yamasa@agri.kagoshima-u.ac.jp).

** Retired the environmental conditions suit citrus production, it is the most important fruit crop grown there. In particular, fruits with good quality can be produced in mid- and late-maturing citrus, such as Ponkan, Tankan, Pummelo, and 'Shiranui', because of the relatively warm autumn and winter. However, over the last four decades there has been a decline in fruit production in this area. To promote citrus consumption, providing information on the health-promoting components of fruit is considered to be very effective. Thus, it is important to elucidate the PMF content of cultivars cultivated in Kagoshima. In addition to large-scale fruit production, there are various local citrus accessions in Kagoshima (Yamamoto et al., 2006, 2011). Although their production is small-scale, these accessions are important as specialized products there. Elucidation of the PMF content of these local accessions is also considered to be important for the citrus industry.

As mentioned above, each citrus accession shows a characteristic PMF content profile (Nogata et al., 2006). However, in major cultivars and local accessions in Kagoshima, although the PMF contents of a few accessions were reported (Nogata et al., 2006; Yamamoto 
et al., 2008), those of several accessions were unclarified. Therefore in the present study, the PMF content of various major cultivated cultivars and local accessions in Kagoshima was quantified. In addition, general fruit traits including the content of ascorbic acid, known as a functional component, were measured.

\section{Materials and Methods}

\section{Plant material}

Bearing trees of 33 citrus accessions grafted on trifoliate orange (Poncirus trifoliata (L.) Raf.) growing in the Toso Orchard of Experimental Farm, Faculty of Agriculture, Kagoshima University (Kagoshima, Japan, ca. $30^{\circ} \mathrm{N}, 131^{\circ} \mathrm{E}$, and $65 \mathrm{~m}$ ) were used (Table 1). A single tree was used for one accession. These accessions were classified into three groups: major cultivars cultivated in Kagoshima, local accessions grown in Kagoshima, and a control.

In local accessions, two shiikuwashas, 'Shiikunin Ama' (10), and 'Shiikunin Kara' (11), could be distinguished from each other by DNA analysis (Yamamoto et al., 2011, 2017). Although fruit traits of 'Yamato \#8'

Table 1. Citrus accessions used in this study.

\begin{tabular}{|c|c|c|c|c|}
\hline No. & Common name & Accession & Latin name & Distribution \\
\hline \multicolumn{5}{|c|}{ Major cultivars cultivated in Kagoshima } \\
\hline \multicolumn{5}{|c|}{ Mandarin and tangor } \\
\hline 1 & Kishu Mikan & Sakurajima Komikan & C. kinokuni hort. ex Tanaka & - \\
\hline 2 & Sastuma mandarin & Okitsu Wase & C. unshiu Marcow. & - \\
\hline 3 & Sastuma mandarin & Otsu 4-gou & C. unshiu Marcow. & - \\
\hline 4 & Ponkan & Yoshida Ponkan & C. reticulata Blanco & - \\
\hline 5 & Ponkan & Sasshu & C. reticulata Blanco & - \\
\hline 6 & Shiranui & Shiranui & $($ C. unshiu $\times$ C. sinensis $) \times C$. reticulata & - \\
\hline 7 & Tankan & Tarumizu 1-gou & C. tankan Hayata & - \\
\hline \multicolumn{5}{|c|}{ Pummelo and its relatives } \\
\hline 8 & Natsumikan & Beni Amanatsu & C. natsudaidai Hayata & - \\
\hline 9 & Pummelo & Otachibana & C. maxima (Burm.) Merr. & - \\
\hline \multicolumn{5}{|c|}{ Local accessions grown in Kagoshima } \\
\hline \multicolumn{5}{|c|}{ Mandarin } \\
\hline 10 & Shiikuwasha & Shiikunin Ama & C. depressa Hayata & Tokunoshima \\
\hline 11 & Shiikuwasha & Shiikunin Kara & C. depressa Hayata & Tokunoshima \\
\hline 12 & & Yamato \#8 & C. depressa relative & Amami Oshima \\
\hline 13 & Kishu Mikan & Usukawa & C. kinokuni hort. ex Tanaka & Kikaijima \\
\hline 14 & Kuroshima Mikan & Shimamikan (Amami) & C. spp. & Amami Oshima \\
\hline 15 & Kuroshima Mikan & Shimamikan (Kuroshima) & C. spp. & Kagoshima \\
\hline 16 & Kuroshima Mikan & Chinazekunin & C. spp. & Tokunoshima \\
\hline 17 & Keraji & Keraji & C. keraji hort. ex Tanaka & Kikaijima \\
\hline 18 & Kabuchii & Kikai Mikan & C. keraji hort. ex Tanaka & Kikaijima \\
\hline 19 & Kabuchii & Natsukunin & C. keraji hort. ex Tanaka & Tokunoshima \\
\hline 20 & Kabuchii & Irabu Oto & C. keraji hort. ex Tanaka & Yoronjima \\
\hline 21 & Kabuchii & Erabumikan & C. keraji hort. ex Tanaka & Kakeromajima \\
\hline 22 & Kabuchii & Kabocha & C. keraji hort. ex Tanaka & Okinoerabujima \\
\hline 23 & Oto & Kurushima & C. oto hort. ex Y. Tanaka & Okinoerabujima \\
\hline 24 & Oto & Yunnu Oto & C. oto hort. ex Y. Tanaka & Yoronjima \\
\hline 25 & Ohto & Ohto & C. spp. & Okinoerabujima \\
\hline 26 & Kunenbo & Tokunin & C. nobilis Lour. & Tokunoshima \\
\hline 27 & Keraji (Kakeroma) & Keraji (Kakeroma) & C. spp. & Kakeromajima \\
\hline 28 & Kimikan & Kimikan & C. flaviculpus hort. ex Tanaka & Kagoshima \\
\hline \multicolumn{5}{|c|}{ Sour orange relatives } \\
\hline 29 & Rokugatsu Mikan & Fusu & C. rokugatsu hort. ex Y. Tanaka & Kikaijima \\
\hline 30 & Shiikuu & Shiikuu & C. spp. & Kikaijima \\
\hline 31 & Shiikuu & Kusa & C. spp. & Amami Oshima \\
\hline 32 & Shiikuu & Tunugekunin & C. spp. & Tokunoshima \\
\hline \multicolumn{5}{|c|}{ Control } \\
\hline 33 & Shiikuwasha & Shiikuwasha & C. depressa Hayata & Okinawa \\
\hline
\end{tabular}


(12) were similar to those of shiikuwasha, isozyme analysis revealed that it was not a true shiikuwasha (Yamamoto et al., 2011). 'Usukawa' (13) has been considered to be a kind of Kishu Mikan based on the morphological traits and DNA profile (Yamamoto, unpublished). Although Kuroshima Mikan (14, 15, and 16) appears to be a Chinese mandarin based on the results of isozyme analysis, its origin has not yet been clarified (Yamamoto et al., 2011). There is a possibility that 'Keraji' (17) originated from Kunenbo and Kabuchii as the seed and pollen parent, respectively (Yamamoto et al., 2010). Kabuchii (18, 19, 20, 21, and 22) may be derived from Kunenbo and Shiikuwasha according to the studies of DNA, isozyme, and chromosome analyses (Yamamoto and Tominaga, 2003; Yamamoto et al., 2010, 2011). The origin of 'Kurushima' (23) and 'Yunnu Oto' (24) is probably similar to that of Kabuchii (Yamamoto et al., 2011). Although 'Ohto' (25) is a relative of Kabuchii, both of them are distinguished clearly (Yamamoto et al., 2011). 'Tokunin' (26) is a true Kunenbo according to the morphological traits and DNA analysis (Yamamoto et al., 2006, 2011). 'Keraji (Kakeroma)' (27) is completely different from ordinary 'Keraji' (17) (Yamamoto et al., 2006, 2011). 'Kimikan' (28) appears to be a relative of yuzu (Yamamoto, unpublished). 'Fusu' (29) is considered to be Rokugatsu Mikan based on the fruit morphology (Yamamoto et al., 2006). Shiikuu (30, 31, and 32) showed genetic influence from Shiikuwasha, and the skin of its fruits has an aroma that is almost the same as that of bergamot (Teramoto et al., 2017). In the control accessions, 'Shiikuwasha' (33) is distributed in Okinawa and it could be distinguished from Shiikuwasha (10 and 11), grown on Tokunoshima, by DNA analysis.

Fruits were sampled from October 5, 2012, to February 6, 2013. The sampling date was determined by the maturing season of each accession. In general, sampling was begun one or two months before fruit maturation. Nine fruits were collected in each sampling. Fruit characteristics (fruit weight, length, and diameter, flesh weight, Brix value, and titratable acidity) of each accession were recorded just after their collection. For measurement of fruit and flesh weight and fruit length and diameter, the data of each of the nine fruits were collected. The Brix value was determined with a digital refractometer (PAL-1; Atago Co., Ltd., Tokyo, Japan). Titratable acidity was measured by titration with $0.156 \mathrm{~N}-\mathrm{NaOH}$ solution. The acidity was expressed as a percentage of citric acid. For analyses of ascorbic acid and PMF, the juice and peel, respectively, were preserved in a freezer $\left(-45^{\circ} \mathrm{C}\right)$. For analyses of the Brix value, titratable acidity, ascorbic acid, and PMF, three fruits were use as one sample. These results were obtained from three replicates.

For analysis of ascorbic acid, the juice was centrifuged $\left(4^{\circ} \mathrm{C}, 10,000 \times \mathrm{g}, 10 \mathrm{~min}\right)$ and the supernatant was used for the assay. The quantity of ascorbic acid was determined by Refrectometer RQflex (Merck, Kenilworth, NJ, USA).

\section{Analysis of polymethoxylated flavones in peel}

In citrus fruit, since most PMF are contained in the peel (Nogata et al., 2006; Yamamoto et al., 2008), only the peel content was measured in the present study. Extraction of PMF from the peel was conducted according to Yoshioka et al. (2001) and Yamamoto et al. (2008). Peel $(1.0 \mathrm{~g} \mathrm{FW})$ was ground in a mortar and extracted in solution (DMSO:MeOH $=1: 1)(30 \mathrm{~mL})$. The solution was centrifuged $(1,400 \times \mathrm{g}, 10 \mathrm{~min})$ and the supernatant was used for the analysis.

The PMF content of the extract was measured by high-performance liquid chromatography (HPLC) (Gulliver system: JASCO, Tokyo, Japan). HPLC analysis was conducted based on the method of Park et al. (1983). The equipment and methods for analysis were as follows; column: Unisil NQC18 $(6.0 \times 100 \mathrm{~mm})$, column temperature: $40^{\circ} \mathrm{C}$, flow rate: $1.2 \mathrm{~mL} \cdot \mathrm{min}^{-1}$, detection wavelength: $340 \mathrm{~nm}(\mathrm{UV})$, mobile phase: solvent A (MeOH:acetonitrile:water $(0.1 \%$ phosphoric acid $)$ (5:10:85)), and solvent $\mathrm{B}$ (MeOH:water $(0.1 \%$ phosphoric acid) (95:5)). The following linear gradient was used: $0 \mathrm{~min}, 100 \% \mathrm{~A}$ and $0 \% \mathrm{~B} ; 45 \mathrm{~min}, 0 \% \mathrm{~A}$ and $100 \%$ B. The major PMF, sinensetin, nobiletin, heptamethoxyflavone, and tangeretin, were quantified.

Shiikuwasha is as an accession bearing fruit with a high PMF content (Inafuku-Teramoto et al., 2010). Thus, in the present study, the PMF content of each accession was evaluated in comparison to the PMF content of 'Shiikuwasha' (33).

\section{Results}

In this study, data of the mature fruits (final sampling) were mainly used for comparison of analyzed fruit characteristics of each cultivar and accession. In addition, data of premature fruits were also examined.

\section{Characteristics of general fruit traits}

Major cultivars in Kagoshima (Table 2): Of the major cultivars, 'Sakurajima Komikan' (1) and 'Otachibana' (9) were the smallest and largest, respectively. In mandarin and tangor, 'Shiranui' (6) was the largest. The percentage of flesh of mandarin and tangor was higher than that of pummelo and its relatives. That of 'Sakurajima Komikan' (1) and Ponkan (4 and 5) was relatively low among mandarin and tangor. The Brix value of all cultivars increased with the maturation of fruits. The value of 'Shiranui' (6) was the highest, followed by 'Tarumizu 1-gou' (7). The titratable acidity of all cultivars except 'Shiranui' (6) and 'Tarumizu 1-gou' (7) decreased with the maturation of fruits. Fruits of 'Tarumizu 1-gou' (7) contained the most ascorbic acid (approx. $40 \mathrm{mg}$ per $100 \mathrm{mLFW}$ ). Its concentration in the remaining cultivars was around $20-30 \mathrm{mg}$ per $100 \mathrm{mLFW}$. 
Table 2. Fruit characteristics of citrus accessions used in this study.

\begin{tabular}{|c|c|c|c|c|c|c|c|c|}
\hline No & Accession & $\begin{array}{l}\text { Sampling date } \\
(\mathrm{y} / \mathrm{m} / \mathrm{d})\end{array}$ & $\begin{array}{l}\text { Fruit weight } \\
(\mathrm{g})\end{array}$ & $\begin{array}{l}\text { Fruit shape } \\
\text { index }\end{array}$ & $\begin{array}{l}\text { Percentage of } \\
\text { flesh }^{y}\end{array}$ & Brix value & $\begin{array}{c}\text { Titratable acidity } \\
(\%)\end{array}$ & $\begin{array}{c}\text { Ascorbic acid } \\
\text { (mg per } 100 \mathrm{mLFW})\end{array}$ \\
\hline \multicolumn{9}{|c|}{ Major cultivars cultivated in Kagoshima } \\
\hline \multicolumn{9}{|c|}{ Mandarin and tangor } \\
\hline \multicolumn{2}{|c|}{1 Sakurajima } & $2012 / 11 / 5$ & $42.5 \pm 1.1^{\mathrm{x}}$ & $145.1 \pm 1.7$ & $77.8 \pm 0.4$ & $10.1 \pm 0.0$ & $1.13 \pm 0.02$ & $23.6 \pm 0.4$ \\
\hline \multirow{3}{*}{\multicolumn{2}{|c|}{$\begin{array}{l}\text { Komikan } \\
2 \text { Okitsu Wase }\end{array}$}} & $2012 / 12 / 5$ & $50.8 \pm 2.1$ & $142.1 \pm 3.0$ & $72.5 \pm 0.5$ & $11.2 \pm 0.1$ & $0.83 \pm 0.01$ & $21.8 \pm 1.2$ \\
\hline & & $2012 / 10 / 5$ & $95.2 \pm 3.2$ & $124.8 \pm 1.4$ & $85.3 \pm 0.3$ & $8.5 \pm 0.1$ & $1.65 \pm 0.04$ & $25.5 \pm 1.3$ \\
\hline & & $2012 / 11 / 5$ & $113.8 \pm 6.0$ & $125.0 \pm 1.6$ & $83.1 \pm 0.7$ & $9.5 \pm 0.2$ & $1.28 \pm 0.03$ & $28.0 \pm 1.6$ \\
\hline \multirow{2}{*}{\multicolumn{2}{|c|}{3 Otsu 4-gou }} & $2012 / 11 / 5$ & $119.4 \pm 1.9$ & $137.1 \pm 2.7$ & $80.2 \pm 0.3$ & $11.4 \pm 0.1$ & $1.41 \pm 0.02$ & $23.8 \pm 0.2$ \\
\hline & & $2012 / 12 / 5$ & $135.9 \pm 6.9$ & $131.2 \pm 2.0$ & $76.3 \pm 1.1$ & $12.5 \pm 0.2$ & $1.30 \pm 0.02$ & $21.8 \pm 0.4$ \\
\hline \multirow{2}{*}{\multicolumn{2}{|c|}{4 Yoshida Ponkan }} & $2012 / 12 / 5$ & $144.3 \pm 5.1$ & $109.5 \pm 1.5$ & $74.9 \pm 1.6$ & $10.5 \pm 0.2$ & $0.88 \pm 0.02$ & $25.7 \pm 0.7$ \\
\hline & & $2013 / 1 / 8$ & $149.5 \pm 1.8$ & $108.1 \pm 1.7$ & $69.9 \pm 0.8$ & $12.6 \pm 0.2$ & $0.78 \pm 0.02$ & $30.8 \pm 0.9$ \\
\hline \multirow{2}{*}{\multicolumn{2}{|c|}{5 Sasshu }} & $2012 / 12 / 5$ & $158.9 \pm 4.1$ & $119.9 \pm 0.9$ & $77.1 \pm 0.7$ & $10.2 \pm 0.0$ & $0.90 \pm 0.02$ & $26.2 \pm 0.7$ \\
\hline & & $2013 / 1 / 8$ & $149.2 \pm 4.8$ & $115.1 \pm 2.3$ & $74.7 \pm 0.7$ & $12.1 \pm 0.2$ & $0.77 \pm 0.03$ & $27.5 \pm 1.1$ \\
\hline \multirow{2}{*}{\multicolumn{2}{|c|}{6 Shiranui }} & $2013 / 1 / 8$ & $288.8 \pm 14.5$ & $101.6 \pm 1.4$ & $80.8 \pm 0.7$ & $12.1 \pm 0.1$ & $1.20 \pm 0.02$ & $33.9 \pm 1.1$ \\
\hline & & $2013 / 2 / 6$ & $245.9 \pm 5.7$ & $101.2 \pm 1.5$ & $82.6 \pm 0.8$ & $14.2 \pm 0.2$ & $1.58 \pm 0.08$ & $28.4 \pm 0.9$ \\
\hline \multirow{2}{*}{\multicolumn{2}{|c|}{7 Tarumizu 1-gou }} & $2013 / 1 / 8$ & $121.3 \pm 6.4$ & $106.4 \pm 0.8$ & $80.4 \pm 0.6$ & $11.3 \pm 0.2$ & $1.30 \pm 0.03$ & $42.9 \pm 1.0$ \\
\hline & & $2013 / 2 / 6$ & $131.7 \pm 4.4$ & $104.7 \pm 0.8$ & $79.4 \pm 0.9$ & $13.0 \pm 0.1$ & $1.36 \pm 0.02$ & $39.9 \pm 1.7$ \\
\hline \multicolumn{9}{|c|}{ Pummelo and its relatives } \\
\hline \multicolumn{2}{|r|}{8 Beni Amanatsu } & $2013 / 2 / 6$ & $340.2 \pm 13.4$ & $123.8 \pm 2.4$ & $69.6 \pm 0.8$ & $8.9 \pm 0.0$ & $1.75 \pm 0.07$ & $24.9 \pm 0.9$ \\
\hline \multicolumn{2}{|c|}{9 Otachibana } & $2013 / 2 / 6$ & $489.0 \pm 15.7$ & $123.5 \pm 1.8$ & $66.7 \pm 2.0$ & $11.0 \pm 0.1$ & $1.40 \pm 0.02$ & $29.5 \pm 2.0$ \\
\hline \multicolumn{9}{|c|}{ Local accessions grown in Kagoshima } \\
\hline \multicolumn{9}{|c|}{ Mandarin } \\
\hline \multirow[t]{3}{*}{10} & Shiikunin Ama & $2012 / 11 / 5$ & $21.7 \pm 0.6$ & $129.2 \pm 3.7$ & $69.9 \pm 0.4$ & $7.8 \pm 0.0$ & $4.86 \pm 0.05$ & $14.4 \pm 0.2$ \\
\hline & & $2012 / 12 / 5$ & $23.6 \pm 0.6$ & $130.7 \pm 1.9$ & $69.5 \pm 0.5$ & $8.6 \pm 0.0$ & $4.07 \pm 0.03$ & $10.8 \pm 1.7$ \\
\hline & & $2013 / 1 / 8$ & $23.0 \pm 0.7$ & $134.9 \pm 2.0$ & $67.1 \pm 0.7$ & $10.5 \pm 0.2$ & $3.66 \pm 0.04$ & $9.4 \pm 0.3$ \\
\hline \multirow[t]{3}{*}{11} & Shiikunin Kara & $2012 / 11 / 5$ & $23.0 \pm 1.0$ & $133.5 \pm 2.3$ & $76.8 \pm 1.5$ & $7.8 \pm 0.1$ & $4.62 \pm 0.55$ & $13.2 \pm 0.4$ \\
\hline & & $2012 / 12 / 5$ & $25.6 \pm 0.8$ & $146.2 \pm 2.3$ & $70.6 \pm 1.0$ & $8.6 \pm 0.1$ & $2.88 \pm 0.09$ & $12.5 \pm 0.2$ \\
\hline & & $2013 / 1 / 8$ & $22.9 \pm 0.8$ & $142.3 \pm 2.3$ & $69.2 \pm 1.1$ & $9.6 \pm 0.1$ & $3.04 \pm 0.01$ & $10.9 \pm 0.3$ \\
\hline \multirow[t]{2}{*}{12} & Yamato \#8 & $2012 / 11 / 5$ & $19.6 \pm 0.7$ & $120.5 \pm 1.5$ & $78.0 \pm 0.6$ & $8.7 \pm 0.1$ & $5.64 \pm 0.15$ & $14.8 \pm 0.3$ \\
\hline & & $2012 / 12 / 5$ & $28.3 \pm 1.1$ & $130.9 \pm 1.5$ & $80.0 \pm 0.4$ & $9.0 \pm 0.1$ & $4.43 \pm 0.03$ & $14.9 \pm 1.0$ \\
\hline 13 & Usukawa & $2012 / 11 / 5$ & $47.2 \pm 3.3$ & $131.9 \pm 2.5$ & $79.6 \pm 0.7$ & $9.2 \pm 0.0$ & $1.01 \pm 0.02$ & $24.9 \pm 0.2$ \\
\hline \multirow[t]{3}{*}{14} & Shimamikan & $2012 / 11 / 5$ & $59.5 \pm 3.2$ & $146.8 \pm 2.0$ & $80.7 \pm 0.4$ & $9.1 \pm 0.0$ & $1.87 \pm 0.09$ & $24.8 \pm 0.9$ \\
\hline & (Amami) & $2012 / 12 / 5$ & $62.1 \pm 2.4$ & $143.3 \pm 1.4$ & $75.5 \pm 0.5$ & $10.6 \pm 0.1$ & $1.11 \pm 0.02$ & $24.2 \pm 1.9$ \\
\hline & & $2013 / 1 / 8$ & $40.6 \pm 2.0$ & $142.0 \pm 2.8$ & $71.6 \pm 0.9$ & $13.8 \pm 0.2$ & $1.11 \pm 0.01$ & $29.9 \pm 0.8$ \\
\hline 15 & Shimamikan & $2012 / 11 / 5$ & $42.2 \pm 1.0$ & $143.4 \pm 1.7$ & $80.6 \pm 0.4$ & $9.1 \pm 0.1$ & $2.27 \pm 0.07$ & $25.3 \pm 0.6$ \\
\hline & (Kuroshima) & $2012 / 12 / 5$ & $55.9 \pm 2.8$ & $151.2 \pm 2.0$ & $76.0 \pm 0.5$ & $10.1 \pm 0.0$ & $1.54 \pm 0.02$ & $28.9 \pm 0.2$ \\
\hline & & $2013 / 1 / 8$ & $45.5 \pm 1.8$ & $149.8 \pm 2.9$ & $72.0 \pm 0.7$ & $12.6 \pm 0.1$ & $1.39 \pm 0.06$ & $32.7 \pm 1.4$ \\
\hline 16 & Chinazekunin & $2012 / 11 / 5$ & $51.7 \pm 1.9$ & $142.6 \pm 2.0$ & $79.1 \pm 0.7$ & $9.2 \pm 0.0$ & $1.88 \pm 0.01$ & $27.3 \pm 1.0$ \\
\hline & & $2012 / 12 / 5$ & $65.6 \pm 2.1$ & $160.8 \pm 1.4$ & $74.0 \pm 0.8$ & $10.2 \pm 0.1$ & $1.34 \pm 0.07$ & $36.9 \pm 1.3$ \\
\hline & & $2013 / 1 / 8$ & $52.2 \pm 2.3$ & $149.1 \pm 3.3$ & $71.3 \pm 0.5$ & $11.5 \pm 0.3$ & $1.14 \pm 0.04$ & $28.7 \pm 2.0$ \\
\hline 17 & Keraji & $2012 / 10 / 5$ & $63.5 \pm 2.9$ & $120.2 \pm 2.7$ & $68.4 \pm 1.0$ & $7.6 \pm 0.1$ & $1.41 \pm 0.03$ & $28.3 \pm 0.2$ \\
\hline & & $2012 / 11 / 5$ & $80.2 \pm 3.4$ & $122.4 \pm 3.4$ & $67.2 \pm 0.6$ & $8.0 \pm 0.0$ & $0.75 \pm 0.01$ & $30.9 \pm 0.4$ \\
\hline & & $2012 / 12 / 5$ & $83.3 \pm 3.9$ & $127.2 \pm 2.3$ & $61.4 \pm 2.6$ & $9.5 \pm 0.0$ & $0.65 \pm 0.01$ & $35.9 \pm 2.3$ \\
\hline 18 & Kikai Mikan & $2012 / 10 / 5$ & $50.0 \pm 2.4$ & $125.2 \pm 1.9$ & $68.4 \pm 1.3$ & $8.1 \pm 0.1$ & $2.78 \pm 0.01$ & $15.8 \pm 0.2$ \\
\hline & & $2012 / 11 / 5$ & $51.8 \pm 2.3$ & $140.6 \pm 4.6$ & $64.6 \pm 0.6$ & $8.2 \pm 0.1$ & $1.44 \pm 0.03$ & $13.7 \pm 1.5$ \\
\hline & & $2012 / 12 / 5$ & $59.4 \pm 1.7$ & $142.2 \pm 4.4$ & $58.2 \pm 0.9$ & $9.0 \pm 0.0$ & $1.13 \pm 0.08$ & $13.5 \pm 1.5$ \\
\hline 19 & Natsukunin & $2012 / 10 / 5$ & $38.6 \pm 2.0$ & $123.2 \pm 2.3$ & $71.8 \pm 0.9$ & $7.4 \pm 0.0$ & $3.08 \pm 0.09$ & $14.3 \pm 0.1$ \\
\hline & & $2012 / 11 / 5$ & $53.4 \pm 2.3$ & $123.0 \pm 4.2$ & $65.4 \pm 0.7$ & $8.0 \pm 0.0$ & $1.68 \pm 0.03$ & $18.3 \pm 1.4$ \\
\hline & & $2012 / 12 / 5$ & $57.1 \pm 3.0$ & $129.6 \pm 2.6$ & $60.7 \pm 0.9$ & $8.4 \pm 0.2$ & $1.32 \pm 0.05$ & $14.7 \pm 1.6$ \\
\hline 20 & Irabu Oto & $2012 / 10 / 5$ & $43.0 \pm 2.3$ & $116.0 \pm 2.4$ & $73.2 \pm 2.8$ & $7.9 \pm 0.0$ & $1.75 \pm 0.08$ & $15.3 \pm 0.1$ \\
\hline & & $2012 / 11 / 5$ & $57.9 \pm 2.6$ & $119.5 \pm 2.2$ & $67.7 \pm 0.8$ & $8.2 \pm 0.1$ & $1.00 \pm 0.03$ & $16.1 \pm 0.9$ \\
\hline & & $2012 / 12 / 5$ & $63.5 \pm 1.9$ & $124.7 \pm 3.2$ & $63.6 \pm 1.1$ & $9.6 \pm 0.1$ & $0.78 \pm 0.02$ & $15.9 \pm 0.5$ \\
\hline 21 & Erabumikan & $2012 / 10 / 5$ & $41.8 \pm 1.5$ & $126.8 \pm 2.5$ & $66.5 \pm 0.8$ & $7.6 \pm 0.0$ & $2.05 \pm 0.07$ & $15.1 \pm 0.5$ \\
\hline & & $2012 / 11 / 5$ & $51.9 \pm 2.2$ & $127.0 \pm 2.4$ & $60.7 \pm 0.8$ & $8.2 \pm 0.1$ & $1.47 \pm 0.08$ & $17.4 \pm 0.3$ \\
\hline
\end{tabular}


Table 2. Continued

\begin{tabular}{|c|c|c|c|c|c|c|c|c|}
\hline No. & Accession & $\begin{array}{l}\text { Sampling date } \\
(y / m / d)\end{array}$ & $\begin{array}{l}\text { Fruit weight } \\
\text { (g) }\end{array}$ & $\begin{array}{l}\text { Fruit shape } \\
\text { index }\end{array}$ & $\begin{array}{l}\text { Percentage of } \\
\text { flesh }^{y}\end{array}$ & Brix value & $\begin{array}{c}\text { Titratable acidity } \\
(\%)\end{array}$ & $\begin{array}{c}\text { Ascorbic acid } \\
\text { (mg per } 100 \mathrm{mLFW})\end{array}$ \\
\hline & & $2012 / 12 / 5$ & $57.2 \pm 1.9$ & $132.1 \pm 2.2$ & $58.4 \pm 2.5$ & $9.0 \pm 0.3$ & $1.17 \pm 0.06$ & $14.9 \pm 1.2$ \\
\hline \multirow[t]{2}{*}{22} & Kabocha & $2012 / 10 / 5$ & $57.8 \pm 3.5$ & $121.5 \pm 4.1$ & $67.3 \pm 0.7$ & $8.2 \pm 0.0$ & $1.83 \pm 0.09$ & $14.8 \pm 1.4$ \\
\hline & & $2012 / 11 / 5$ & $74.6 \pm 2.5$ & $126.2 \pm 1.8$ & $63.7 \pm 2.3$ & $8.6 \pm 0.1$ & $1.15 \pm 0.01$ & $17.7 \pm 1.3$ \\
\hline \multirow[t]{3}{*}{23} & Kurushima & $2012 / 11 / 5$ & $59.2 \pm 1.9$ & $122.8 \pm 1.8$ & $81.3 \pm 0.3$ & $9.0 \pm 0.0$ & $2.20 \pm 0.01$ & $12.6 \pm 0.3$ \\
\hline & & $2012 / 12 / 5$ & $63.9 \pm 1.7$ & $121.3 \pm 1.5$ & $81.5 \pm 0.4$ & $10.1 \pm 0.09$ & $2.20 \pm 0.04$ & $17.9 \pm 0.4$ \\
\hline & & $2013 / 1 / 8$ & $56.8 \pm 2.0$ & $120.7 \pm 1.7$ & $81.7 \pm 0.6$ & $11.2 \pm 0.0$ & $1.98 \pm 0.02$ & $20.1 \pm 1.2$ \\
\hline \multirow[t]{3}{*}{24} & Yunnu Oto & $2012 / 11 / 5$ & $57.3 \pm 1.7$ & $117.1 \pm 7.1$ & $75.7 \pm 1.6$ & $8.4 \pm 0.1$ & $2.36 \pm 0.02$ & $9.8 \pm 0.7$ \\
\hline & & $2012 / 12 / 5$ & $61.1 \pm 3.4$ & $125.0 \pm 1.5$ & $74.5 \pm 1.5$ & $9.8 \pm 0.0$ & $1.97 \pm 0.02$ & $14.9 \pm 1.0$ \\
\hline & & $2013 / 1 / 8$ & $53.8 \pm 1.3$ & $131.4 \pm 1.1$ & $74.9 \pm 0.6$ & $10.9 \pm 0.2$ & $1.87 \pm 0.05$ & $16.6 \pm 0.4$ \\
\hline & Otoh & $2012 / 11 / 5$ & $54.1 \pm 2.6$ & $134.8 \pm 2.6$ & $68.6 \pm 1.3$ & $8.1 \pm 0.2$ & $1.36 \pm 0.03$ & $11.5 \pm 0.9$ \\
\hline & & $2012 / 12 / 5$ & $47.0 \pm 2.8$ & $123.8 \pm 4.6$ & $63.1 \pm 1.4$ & $8.4 \pm 0.1$ & $1.24 \pm 0.03$ & $11.3 \pm 0.9$ \\
\hline & & $2013 / 1 / 8$ & $37.0 \pm 1.1$ & $127.2 \pm 3.1$ & $60.7 \pm 1.9$ & $8.8 \pm 0.1$ & $1.15 \pm 0.03$ & $9.4 \pm 0.3$ \\
\hline \multirow[t]{2}{*}{26} & Tokunin & $2012 / 12 / 5$ & $170.4 \pm 8.1$ & $132.0 \pm 9.0$ & $70.6 \pm 0.8$ & $8.1 \pm 0.1$ & $2.21 \pm 0.05$ & $21.6 \pm 0.7$ \\
\hline & & $2013 / 1 / 8$ & $181.0 \pm 3.5$ & $131.4 \pm 1.2$ & $70.4 \pm 1.2$ & $9.0 \pm 0.0$ & $1.92 \pm 0.01$ & $27.3 \pm 1.2$ \\
\hline \multirow[t]{2}{*}{27} & Keraji & $2013 / 1 / 8$ & $83.7 \pm 5.3$ & $133.7 \pm 1.5$ & $63.1 \pm 1.2$ & $10.8 \pm 0.1$ & $2.05 \pm 0.08$ & $20.0 \pm 1.1$ \\
\hline & (Kakeroma) & $2013 / 2 / 6$ & $83.5 \pm 4.0$ & $129.1 \pm 2.0$ & $63.2 \pm 1.5$ & $12.5 \pm 0.1$ & $1.81 \pm 0.06$ & $10.9 \pm 3.5$ \\
\hline \multirow[t]{2}{*}{28} & Kimikan & $2013 / 1 / 8$ & $95.2 \pm 5.7$ & $111.2 \pm 1.1$ & $75.4 \pm 0.6$ & $11.1 \pm 0.1$ & $2.06 \pm 0.03$ & $29.8 \pm 1.3$ \\
\hline & & $2013 / 2 / 6$ & $99.5 \pm 5.1$ & $109.0 \pm 1.4$ & $75.4 \pm 0.5$ & $12.4 \pm 0.1$ & $1.96 \pm 0.03$ & $28.5 \pm 4.5$ \\
\hline \multicolumn{9}{|c|}{ Sour orange relatives } \\
\hline \multirow[t]{2}{*}{29} & Fusu & $2013 / 1 / 8$ & $89.1 \pm 3.2$ & $126.6 \pm 2.3$ & $67.1 \pm 0.8$ & $8.9 \pm 0.0$ & $2.93 \pm 0.05$ & $40.6 \pm 2.4$ \\
\hline & & $2013 / 2 / 6$ & $90.4 \pm 4.4$ & $132.3 \pm 1.9$ & $64.6 \pm 0.7$ & $10.6 \pm 0.1$ & $2.51 \pm 0.03$ & $42.6 \pm 0.3$ \\
\hline \multirow[t]{2}{*}{30} & Shiikuu & $2013 / 1 / 8$ & $38.2 \pm 1.5$ & $114.0 \pm 1.7$ & $63.1 \pm 1.2$ & $8.7 \pm 0.1$ & $3.02 \pm 0.04$ & $8.7 \pm 0.2$ \\
\hline & & $2013 / 2 / 6$ & $43.8 \pm 1.5$ & $118.3 \pm 1.0$ & $61.3 \pm 0.6$ & $11.2 \pm 0.1$ & $2.76 \pm 0.08$ & $11.8 \pm 1.4$ \\
\hline \multirow[t]{2}{*}{31} & Kusa & $2013 / 1 / 8$ & $41.4 \pm 1.5$ & $115.9 \pm 1.4$ & $58.7 \pm 0.5$ & $8.3 \pm 0.1$ & $2.31 \pm 0.05$ & $12.5 \pm 1.2$ \\
\hline & & $2013 / 2 / 6$ & $48.1 \pm 2.3$ & $117.6 \pm 1.7$ & $57.1 \pm 1.0$ & $11.1 \pm 0.0$ & $2.18 \pm 0.07$ & $14.3 \pm 4.6$ \\
\hline \multirow[t]{2}{*}{32} & Tunugekunin & $2013 / 1 / 8$ & $48.4 \pm 1.1$ & $121.9 \pm 1.3$ & $65.1 \pm 0.8$ & $8.6 \pm 0.2$ & $2.83 \pm 0.06$ & $9.5 \pm 0.8$ \\
\hline & & $2013 / 2 / 6$ & $52.0 \pm 2.1$ & $125.5 \pm 1.7$ & $62.9 \pm 0.9$ & $10.8 \pm 0.1$ & $2.64 \pm 0.03$ & $11.4 \pm 3.6$ \\
\hline \multicolumn{9}{|c|}{ Control } \\
\hline \multirow[t]{3}{*}{33} & Shiikuwasha & $2012 / 11 / 5$ & $19.4 \pm 1.0$ & $131.0 \pm 2.1$ & $73.9 \pm 0.5$ & $7.6 \pm 0.1$ & $3.66 \pm 0.13$ & $15.0 \pm 1.3$ \\
\hline & & $2012 / 12 / 5$ & $24.8 \pm 0.6$ & $143.6 \pm 2.6$ & $69.3 \pm 0.5$ & $8.7 \pm 0.1$ & $2.73 \pm 0.05$ & $14.8 \pm 0.8$ \\
\hline & & $2013 / 1 / 8$ & $20.2 \pm 0.8$ & $132.6 \pm 2.4$ & $67.7 \pm 0.7$ & $9.9 \pm 0.1$ & $2.89 \pm 0.03$ & $12.5 \pm 0.8$ \\
\hline
\end{tabular}

${ }^{\mathrm{z}}$ Fruit diameter/fruit length $\times 100$.

${ }^{y}$ Flesh weight/fruit weight $\times 100$.

${ }^{x}$ Standard error.

Local accessions in Kagoshima (Table 2): In general, fruits of the local accessions were small. In particular, Shiikuwasha (10 and 11) fruit was very small. Only 'Tokunin' (26) bore fruit larger than $100 \mathrm{~g}$. The percentage of flesh of local accessions tended to be lower than that of the major cultivars. That of 'Keraji' (17), Kabuchii (18, 19, 20, 21, and 22), Ohto (25), and sour orange relatives $(29,30,31$, and 32$)$ was lower by approximately $70 \%$. Although the Brix value of many local accessions was low, fruit of 'Shimamikan' (14 and $15)$ showed a relatively high value. The titratable acidity of 'Keraji' (17) was very low. In contrast, that of Shiikuwasha (10 and 11) and its relative 'Yamato \#8' (12) was high. The ascorbic acid level of 'Fusu' (29) and 'Keraji' (17) was the highest and the second highest, respectively. That of Shiikuwasha (10 and 11) and 'Yamato \#8' (12), Kabuchii (18, 19, 20, 21, and 22), 'Ohto' (25), and Shiikuu (30, 31, and 32) was low.

\section{Polymethoxylated flavones content of peel}

In all the accessions used in the present study, the peel contained PMF. Nobiletin and tangeretin were the major PMF among the four PMF components analyzed. In Satsuma mandarin (2 and 3), 'Keraji' (17), and Oto (23 and 24), a relatively high percentage of heptamethoxyflavone was observed. Heptamethoxyflavone was not detected in Kishu Mikan (1 and 13), Ponkan (4 and 5), 'Shiranui' (6), 'Otachibana' (9), Shiikuwasha $(10,11$, and 33) or its relative 'Yamato \#8' (12), Kuroshima Mikan (14, 15, and 16), 'Ohto' (25), 'Keraji (Kakeroma)' (27), 'Kimikan' (28), or Shiikuu (30, 31, and 32). Shinensetin was not detected in Satsuma mandarin (2 and 3), 'Beni Amanatsu' (8), 'Otachibana' (9), or 'Tokunin' (26). Nobiletin was only detected in 'Otachibana' (9) (Table 3).

Seasonal changes in the PMF content were not clear. The levels in many accessions were mostly stable dur- 
Table 3. Contents of polymethoxylated flavones of peel in citrus accessions used in this study.

\begin{tabular}{|c|c|c|c|c|c|c|c|}
\hline \multirow{2}{*}{ No } & \multirow{2}{*}{ Accession } & \multirow{2}{*}{$\begin{array}{l}\text { Sampling date } \\
(\mathrm{y} / \mathrm{m} / \mathrm{d})\end{array}$} & \multicolumn{5}{|c|}{ Polymethoxylated flavones content ${ }^{2}\left(\mu \mathrm{g} \cdot \mathrm{gFW}^{-1}\right)$} \\
\hline & & & SIN & NOB & HMF & TAN & Total \\
\hline \multicolumn{8}{|c|}{ Major cultivars cultivated in Kagoshima } \\
\hline \multicolumn{8}{|c|}{ Mandarin and tangor } \\
\hline \multirow[t]{2}{*}{1} & Sakurajima & $2012 / 11 / 5$ & $137.9 \pm 15.8^{\mathrm{y}}$ & $1234.9 \pm 116.2$ & n.d. ${ }^{x}$ & $955.1 \pm 121.4$ & $2327.9 \pm 251.8$ \\
\hline & Komikan & $2012 / 12 / 5$ & $108.3 \pm 19.7$ & $1045.5 \pm 199.0$ & n.d. & $919.2 \pm 168.8$ & $2073.0 \pm 387.4$ \\
\hline \multirow[t]{2}{*}{2} & Okitsu Wase & $2012 / 10 / 5$ & n.d. & $119.2 \pm 13.3$ & $84.2 \pm 4.2$ & $92.2 \pm 12.9$ & $295.6 \pm 21.5$ \\
\hline & & $2012 / 11 / 5$ & n.d. & $126.4 \pm 15.7$ & $74.9 \pm 5.0$ & $91.4 \pm 13.3$ & $292.7 \pm 33.1$ \\
\hline \multirow[t]{2}{*}{3} & Otsu 4-gou & $2012 / 11 / 5$ & n.d. & $94.2 \pm 12.8$ & $57.6 \pm 7.3$ & $51.0 \pm 7.3$ & $202.8 \pm 14.7$ \\
\hline & & $2012 / 12 / 5$ & n.d. & $130.7 \pm 21.8$ & $79.3 \pm 4.1$ & $95.8 \pm 17.3$ & $305.8 \pm 42.6$ \\
\hline \multirow[t]{2}{*}{4} & Yoshida Ponkan & $2012 / 12 / 5$ & $112.9 \pm 14.5$ & $1203.4 \pm 141.8$ & n.d. & $1576.1 \pm 206.8$ & $2892.4 \pm 362.7$ \\
\hline & & $2013 / 1 / 8$ & $93.4 \pm 9.9$ & $1052.0 \pm 107.1$ & n.d. & $1481.9 \pm 142.0$ & $2627.3 \pm 257.1$ \\
\hline \multirow[t]{2}{*}{5} & Sasshu & $2012 / 12 / 5$ & $129.3 \pm 9.8$ & $1295.3 \pm 65.5$ & n.d. & $1769.7 \pm 152.6$ & $3194.3 \pm 213.9$ \\
\hline & & $2013 / 1 / 8$ & $92.6 \pm 8.1$ & $1024.8 \pm 46.0$ & n.d. & $1510.0 \pm 50.4$ & $2627.4 \pm 101.9$ \\
\hline \multirow[t]{2}{*}{6} & Shiranui & $2013 / 1 / 8$ & $95.2 \pm 20.9$ & $408.2 \pm 73.0$ & n.d. & $122.5 \pm 16.5$ & $625.9 \pm 110.1$ \\
\hline & & $2013 / 2 / 6$ & $230.8 \pm 42.7$ & $721.7 \pm 84.0$ & n.d. & $153.7 \pm 16.6$ & $1106.2 \pm 135.8$ \\
\hline \multirow[t]{2}{*}{7} & Tarumizu 1-gou & $2013 / 1 / 8$ & $160.0 \pm 2.2$ & $1318.0 \pm 14.5$ & $116.8 \pm 4.6$ & $709.5 \pm 31.6$ & $2304.3 \pm 41.6$ \\
\hline & & $2013 / 2 / 6$ & $201.9 \pm 7.8$ & $1628.1 \pm 43.8$ & $138.1 \pm 5.9$ & $927.9 \pm 30.6$ & $2896.0 \pm 59.0$ \\
\hline \multicolumn{8}{|c|}{ Pummelo and its relatives } \\
\hline 8 & Beni Amanatsu & $2013 / 2 / 6$ & n.d. & $122.0 \pm 25.6$ & $20.0 \pm 0.0$ & $82.0 \pm 4.4$ & $224.0 \pm 26.6$ \\
\hline 9 & Otachibana & $2013 / 2 / 6$ & n.d. & $60.6 \pm 1.4$ & n.d. & n.d. & $60.6 \pm 1.4$ \\
\hline \multicolumn{8}{|c|}{ Local accessions grown in Kagoshima } \\
\hline \multicolumn{8}{|c|}{ Mandarin } \\
\hline \multirow[t]{3}{*}{10} & Shiikunin Ama & $2012 / 11 / 5$ & $134.6 \pm 2.4$ & $1264.2 \pm 12.7$ & n.d. & $1042.4 \pm 14.5$ & $2441.2 \pm 19.9$ \\
\hline & & $2012 / 12 / 5$ & $165.4 \pm 11.9$ & $1540.3 \pm 120.6$ & n.d. & $1528.4 \pm 145.7$ & $3234.1 \pm 276.3$ \\
\hline & & $2013 / 1 / 8$ & $192.1 \pm 6.3$ & $1747.0 \pm 62.8$ & n.d. & $1755.9 \pm 109.0$ & $3695.0 \pm 175.9$ \\
\hline \multirow[t]{3}{*}{11} & Shiikunin Kara & $2012 / 11 / 5$ & $103.2 \pm 7.8$ & $1375.2 \pm 102.1$ & n.d. & $978.6 \pm 80.3$ & $2457.0 \pm 184.6$ \\
\hline & & $2012 / 12 / 5$ & $97.4 \pm 1.9$ & $1265.5 \pm 34.8$ & n.d. & $979.5 \pm 53.2$ & $2342.5 \pm 89.5$ \\
\hline & & $2013 / 1 / 8$ & $101.6 \pm 11.4$ & $1326.0 \pm 129.9$ & n.d. & $1063.3 \pm 130.6$ & $2490.9 \pm 271.8$ \\
\hline 12 & Yamato \#8 & $2012 / 11 / 5$ & $93.9 \pm 3.4$ & $1141.9 \pm 58.9$ & n.d. & $692.4 \pm 35.6$ & $1928.1 \pm 94.8$ \\
\hline & & $2012 / 12 / 5$ & $101.7 \pm 9.9$ & $1226.9 \pm 112.7$ & n.d. & $909.6 \pm 88.2$ & $2238.2 \pm 210.7$ \\
\hline 13 & Usukawa & $2012 / 11 / 5$ & $154.5 \pm 7.5$ & $1621.8 \pm 127.5$ & n.d. & $1495.0 \pm 193.6$ & $3271.3 \pm 328.1$ \\
\hline 14 & Shimamikan & $2012 / 11 / 5$ & $157.9 \pm 16.1$ & $737.8 \pm 31.0$ & n.d. & $997.2 \pm 21.3$ & $1892.9 \pm 45.1$ \\
\hline & (Amami) & $2012 / 12 / 5$ & $175.1 \pm 18.9$ & $743.2 \pm 90.4$ & n.d. & $1103.7 \pm 129.7$ & $2021.9 \pm 238.8$ \\
\hline & & $2013 / 1 / 8$ & $220.8 \pm 23.8$ & $780.2 \pm 9.9$ & n.d. & $1229.9 \pm 49.1$ & $2230.9 \pm 80.0$ \\
\hline 15 & Shimamikan & $2012 / 11 / 5$ & $159.5 \pm 19.6$ & $656.4 \pm 50.7$ & n.d. & $937.3 \pm 60.8$ & $1753.2 \pm 130.7$ \\
\hline & (Kuroshima) & $2012 / 12 / 5$ & n.d. & $589.2 \pm 33.3$ & n.d. & $954.7 \pm 56.7$ & $1543.9 \pm 89.9$ \\
\hline & & $2013 / 1 / 8$ & $159.8 \pm 12.2$ & $611.7 \pm 33.1$ & n.d. & $1043.8 \pm 82.1$ & $1815.3 \pm 126.5$ \\
\hline 16 & Chinazekunin & $2012 / 11 / 5$ & $180.5 \pm 30.9$ & $698.7 \pm 144.1$ & n.d. & $874.8 \pm 189.9$ & $1754.0 \pm 364.8$ \\
\hline & & $2012 / 12 / 5$ & n.d. & $580.4 \pm 60.6$ & n.d. & $815.7 \pm 92.2$ & $1396.1 \pm 152.8$ \\
\hline & & $2013 / 1 / 8$ & $153.3 \pm 19.7$ & $538.5 \pm 92.0$ & n.d. & $788.5 \pm 120.8$ & $1480.3 \pm 231.7$ \\
\hline 17 & Keraji & $2012 / 10 / 5$ & n.d. & $293.8 \pm 17.5$ & $158.0 \pm 9.2$ & $317.0 \pm 27.1$ & $768.8 \pm 51.1$ \\
\hline & & $2012 / 11 / 5$ & $109.5 \pm 21.5$ & $188.5 \pm 28.9$ & $106.7 \pm 12.8$ & $220.1 \pm 22.7$ & $624.8 \pm 82.8$ \\
\hline & & $2012 / 12 / 5$ & n.d. & $211.1 \pm 19.9$ & $105.6 \pm 12.8$ & $262.9 \pm 19.1$ & $579.6 \pm 51.0$ \\
\hline 18 & Kikai Mikan & $2012 / 10 / 5$ & $51.9 \pm 6.3$ & $767.5 \pm 67.1$ & $213.4 \pm 25.5$ & $1192.6 \pm 119.5$ & $2225.4 \pm 218.1$ \\
\hline & & $2012 / 11 / 5$ & $42.7 \pm 5.2$ & $476.4 \pm 77.6$ & $110.7 \pm 14.9$ & $738.9 \pm 120.6$ & $1368.7 \pm 217.1$ \\
\hline & & $2012 / 12 / 5$ & $39.3 \pm 2.3$ & $441.4 \pm 20.5$ & $110.2 \pm 2.0$ & $843.1 \pm 14.0$ & $1434.0 \pm 35.9$ \\
\hline 19 & Natsukunin & $2012 / 10 / 5$ & $42.9 \pm 1.5$ & $647.6 \pm 11.2$ & $143.1 \pm 4.1$ & $1012.4 \pm 18.2$ & $1846.0 \pm 25.9$ \\
\hline & & $2012 / 11 / 5$ & $36.4 \pm 2.4$ & $500.1 \pm 45.8$ & $105.8 \pm 14.3$ & $793.8 \pm 92.7$ & $1436.1 \pm 154.7$ \\
\hline & & $2012 / 12 / 5$ & $38.3 \pm 3.8$ & $461.4 \pm 58.7$ & $110.5 \pm 18.1$ & $879.1 \pm 129.6$ & $1489.3 \pm 209.9$ \\
\hline 20 & Irabu Oto & $2012 / 10 / 5$ & $22.2 \pm 2.3$ & $338.5 \pm 38.2$ & $68.6 \pm 10.2$ & $481.4 \pm 37.7$ & $910.7 \pm 87.9$ \\
\hline & & $2012 / 11 / 5$ & $45.8 \pm 5.5$ & $555.8 \pm 47.4$ & $112.1 \pm 10.5$ & $963.1 \pm 73.8$ & $1676.8 \pm 135.9$ \\
\hline & & $2012 / 12 / 5$ & $37.3 \pm 3.7$ & $382.7 \pm 49.2$ & $85.6 \pm 11.0$ & $704.5 \pm 78.3$ & $1210.1 \pm 142.0$ \\
\hline 21 & Erabumikan & $2012 / 10 / 5$ & $39.6 \pm 2.4$ & $614.4 \pm 36.8$ & $151.0 \pm 15.1$ & $872.1 \pm 71.7$ & $1677.1 \pm 125.7$ \\
\hline
\end{tabular}


Table 3. Continued

\begin{tabular}{|c|c|c|c|c|c|c|c|}
\hline \multirow{2}{*}{ No. } & \multirow{2}{*}{ Accession } & \multirow{2}{*}{$\begin{array}{l}\text { Sampling date } \\
(\mathrm{y} / \mathrm{m} / \mathrm{d})\end{array}$} & \multicolumn{5}{|c|}{ Polymethoxylated flavones content ${ }^{\mathrm{z}}\left(\mu \mathrm{g} \cdot \mathrm{gFW}^{-1}\right)$} \\
\hline & & & SIN & NOB & HMF & TAN & Total \\
\hline & & $2012 / 11 / 5$ & $33.7 \pm 1.7$ & $422.9 \pm 18.7$ & $97.3 \pm 2.9$ & $727.2 \pm 10.1$ & $1281.1 \pm 29.8$ \\
\hline & & $2012 / 12 / 5$ & $36.9 \pm 2.7$ & $420.6 \pm 31.0$ & $96.8 \pm 7.3$ & $748.7 \pm 44.5$ & $1303.0 \pm 85.2$ \\
\hline \multirow[t]{2}{*}{22} & Kabocha & $2012 / 10 / 5$ & $32.9 \pm 4.4$ & $439.2 \pm 40.9$ & $86.3 \pm 15.3$ & $645.8 \pm 99.4$ & $1204.2 \pm 158.1$ \\
\hline & & $2012 / 11 / 5$ & $37.1 \pm 1.2$ & $394.0 \pm 9.6$ & $62.9 \pm 4.1$ & $575.0 \pm 24.2$ & $1069.0 \pm 33.8$ \\
\hline \multirow[t]{3}{*}{23} & Kurushima & $2012 / 11 / 5$ & $22.6 \pm 0.7$ & $199.4 \pm 1.5$ & $167.7 \pm 12.5$ & $180.0 \pm 9.1$ & $569.7 \pm 21.8$ \\
\hline & & $2012 / 12 / 5$ & $27.2 \pm 3.3$ & $215.6 \pm 26.2$ & $204.1 \pm 39.2$ & $228.2 \pm 34.4$ & $675.1 \pm 102.6$ \\
\hline & & $2013 / 1 / 8$ & $35.9 \pm 1.5$ & $277.7 \pm 16.9$ & $266.9 \pm 31.5$ & $310.7 \pm 30.1$ & $891.2 \pm 79.6$ \\
\hline \multirow[t]{3}{*}{24} & Yunnu Oto & $2012 / 11 / 5$ & $25.1 \pm 2.4$ & $189.9 \pm 9.6$ & $212.8 \pm 20.9$ & $176.5 \pm 12.6$ & $604.3 \pm 44.9$ \\
\hline & & $2012 / 12 / 5$ & $24.7 \pm 2.2$ & $179.0 \pm 14.8$ & $204.3 \pm 18.2$ & $173.2 \pm 16.6$ & $581.2 \pm 51.6$ \\
\hline & & $2013 / 1 / 8$ & $32.2 \pm 1.5$ & $253.9 \pm 8.1$ & $250.9 \pm 18.8$ & $263.4 \pm 19.4$ & $800.4 \pm 43.4$ \\
\hline \multirow[t]{3}{*}{25} & Ohto & $2012 / 11 / 5$ & $40.7 \pm 4.1$ & $358.4 \pm 41.0$ & n.d. & $329.5 \pm 23.8$ & $728.6 \pm 68.9$ \\
\hline & & $2012 / 12 / 5$ & $35.3 \pm 3.7$ & $361.7 \pm 33.8$ & n.d. & $438.0 \pm 49.1$ & $835.0 \pm 86.3$ \\
\hline & & $2013 / 1 / 8$ & $52.5 \pm 7.9$ & $560.3 \pm 80.5$ & n.d. & $636.7 \pm 66.3$ & $1249.5 \pm 154.6$ \\
\hline \multirow[t]{2}{*}{26} & Tokunin & $2012 / 12 / 5$ & n.d. & $71.4 \pm 1.0$ & $104.8 \pm 7.5$ & $78.1 \pm 1.7$ & $254.3 \pm 7.8$ \\
\hline & & $2013 / 1 / 8$ & n.d. & $107.4 \pm 4.7$ & $120.6 \pm 6.2$ & $104.8 \pm 1.8$ & $332.8 \pm 2.4$ \\
\hline \multirow[t]{2}{*}{27} & Keraji & $2013 / 1 / 8$ & $85.1 \pm 11.5$ & $468.2 \pm 52.3$ & n.d. & $271.4 \pm 39.0$ & $824.7 \pm 95.4$ \\
\hline & (Kakeroma) & $2013 / 2 / 6$ & $86.6 \pm 5.1$ & $475.8 \pm 11.4$ & n.d. & $275.1 \pm 15.8$ & $837.5 \pm 2.7$ \\
\hline \multirow[t]{2}{*}{28} & Kimikan & $2013 / 1 / 8$ & $32.1 \pm 0.4$ & $492.5 \pm 13.4$ & n.d. & $449.1 \pm 6.0$ & $973.7 \pm 17.2$ \\
\hline & & $2013 / 2 / 6$ & $40.4 \pm 7.1$ & $599.9 \pm 87.1$ & n.d. & $547.1 \pm 64.8$ & $1187.4 \pm 158.9$ \\
\hline \multicolumn{8}{|c|}{ Sour orange relatives } \\
\hline \multirow[t]{2}{*}{29} & Fusu & $2013 / 1 / 8$ & $44.5 \pm 5.8$ & $397.0 \pm 43.3$ & $60.4 \pm 12.6$ & $119.1 \pm 9.3$ & $621.0 \pm 65.4$ \\
\hline & & $2013 / 2 / 6$ & $30.2 \pm 3.7$ & $260.8 \pm 20.1$ & $39.7 \pm 3.5$ & $57.0 \pm 3.9$ & $387.7 \pm 31.2$ \\
\hline \multirow[t]{2}{*}{30} & Shiikuu & $2013 / 1 / 8$ & $64.2 \pm 4.1$ & $321.0 \pm 19.3$ & n.d. & $104.1 \pm 5.6$ & $489.3 \pm 28.8$ \\
\hline & & $2013 / 2 / 6$ & $71.2 \pm 1.9$ & $361.0 \pm 5.4$ & n.d. & $97.6 \pm 2.8$ & $529.8 \pm 4.8$ \\
\hline \multirow[t]{2}{*}{31} & Kusa & $2013 / 1 / 8$ & $72.2 \pm 5.0$ & $355.6 \pm 21.5$ & n.d. & $116.5 \pm 6.4$ & $544.3 \pm 32.2$ \\
\hline & & $2013 / 2 / 6$ & $68.4 \pm 2.0$ & $361.6 \pm 8.4$ & n.d. & $120.1 \pm 5.4$ & $550.1 \pm 13.7$ \\
\hline \multirow[t]{2}{*}{32} & Tunugekunin & $2013 / 1 / 8$ & $84.7 \pm 7.5$ & $409.0 \pm 34.4$ & n.d. & $133.0 \pm 14.7$ & $626.7 \pm 56.2$ \\
\hline & & $2013 / 2 / 6$ & $84.4 \pm 2.6$ & $419.1 \pm 4.2$ & n.d. & $130.3 \pm 5.4$ & $633.8 \pm 5.2$ \\
\hline \multicolumn{8}{|c|}{ Control } \\
\hline \multirow[t]{3}{*}{33} & Shiikuwasha & $2012 / 11 / 5$ & $89.5 \pm 1.4$ & $1247.7 \pm 23.9$ & n.d. & $855.5 \pm 13.7$ & $2192.7 \pm 36.1$ \\
\hline & & $2012 / 12 / 5$ & $75.8 \pm 0.9$ & $1016.5 \pm 15.7$ & n.d. & $799.7 \pm 19.1$ & $1892.0 \pm 29.4$ \\
\hline & & $2013 / 1 / 8$ & $82.8 \pm 8.5$ & $1112.1 \pm 110.1$ & n.d. & $872.2 \pm 78.5$ & $2067.1 \pm 196.8$ \\
\hline
\end{tabular}

z SIN: sinensetin, NOB: nobiletin, HMF: heptamethoxyflavone, TAN: tangeretin.

${ }^{y}$ Standard error.

${ }^{x}$ n.d.: Not detected.

ing the study. However, in some accessions, there were differences in the PMF content between premature and mature fruits. The contents of mature fruits were higher than those of premature fruits in 'Shiranui' (6), 'Shiikunin Ama' (10), and 'Ohto' (25). In contrast, the contents of premature fruits were higher than those of mature fruits in 'Kikai Mikan' (18), 'Natsukunin' (19), 'Erabumikan' (21), and 'Fusu' (29) (Table 3).

Major cultivars in Kagoshima (Table 3): The PMF contents of 'Sakurajima Komikan' (1), Ponkan (4 and 5), and 'Tarumizu 1-gou' (7) were almost the same as or more than that of the control 'Shiikuwasha' (33). The contents of Satsuma mandarin (2 and 3), 'Beni Amanatsu' (8), and 'Otachibana' (9) were very low.

Local accessions in Kagoshima (Table 3): The PMF contents of Shiikuwasha accessions grown on
Tokunoshima (10 and 11) and its relative 'Yamato \#8' (12) were high. 'Usukawa' (13) also showed a high PMF content. The contents in Kuroshima Mikan (14, 15, and 16) and Kabuchii $(18,19,20,21$, and 22) were almost the same as or somewhat lower than that in 'Shiikuwasha' (33). 'Tokunin' (26) had the lowest PMF content.

\section{Discussion}

The present study revealed accession differences in the PMF content and composition of fruit (peel) in major cultivars and local accessions of Kagoshima. The PMF contents of accessions belonging to the same species (common name) were almost the same. Thus, the accession differences are considered to be derived from genetic differences, although only one tree was used for 
each accession. In addition, as shown in Table 2, since all samples showed typical fruit traits of each cultivar and accession, our PMF data is considered to be reliable.

Although seasonal changes in the PMF content were not clear in general, the content of some accessions such as 'Irabu Oto' (20) fluctuated. In addition, sinensetin was not detected in some accessions $(15,16,17)$ in one or two of the three analyses. The possibility that these fluctuations were caused by analysis error could not be ruled out. However, this point could not be clarified in the present study.

Nobiletin and tangeretin were the most abundant PMF in many accessions used in the present study. These results are consistent with those of a previous study (Nogata et al., 2006). Green et al. (2007) and Inafuku-Teramoto et al. (2011) reported that heptamethoxyflavone is a major component as well as nobiletin and tangeretin. In the present study also, the percentage of heptamethoxyflavone was relatively high in Satsuma mandarin (2 and 3), 'Keraji' (17), and Oto (23 and 24). However, heptamethoxyflavone was not detected in Kishu Mikan (1 and 13), Ponkan (4 and 5), 'Shiranui' (6), 'Otachibana' (9), Shiikuwasha (10, 11, and 33), and its relative (12), Kuroshima Mikan (14, 15, and 16), 'Ohto' (25), 'Keraji (Kakeroma)' (27), 'Kimikan' (28), or Shiikuu (30, 31 and 32). Clear cultivar or accession differences existed in the composition of components of PMF. In all cultivars and accessions analyzed, sinensetin was not found to be a major PMF.

On comparison with the control 'Shiikuwasha' (33), which originated in Okinawa and has a high PMF level, the PMF content in Kishu Mikan (1 and 13), Ponkan (4 and 5), 'Tarumizu 1-gou' (7), and Shiikuwasha (10 and 11), which are distributed in Kagoshima, were high. Kuroshima Mikan (14, 15, and 16) and Kabuchii (18, 19, 20, 21, and 22) also showed relatively high PMF contents. These results are consistent with previous studies that investigated the PMF contents of Ponkan, Tankan, Shiikuwasha, and Kabuchii (Inafuku-Teramoto, 2010, 2011; Nogata et al., 2006; Yamamoto et al., 2008). To our knowledge, this is the first report of a high PMF content in Kishu Mikan and Kuroshima Mikan. In contrast, the PMF content in Sastuma mandarin (2 and 3), 'Beni Amanatsu' (8), 'Otachibana' (9), and 'Tokunin' (Kunenbo) (26) was very low. These results agree with those of Nogata et al. (2006), who analyzed Satsuma mandarin and pummelo and its relative. However, the results for Kunenbo were different from those of Inafuku-Teramoto et al. (2011). Materials used in both studies were the same species but different accessions. This may be one reason for the difference in their content. PMF contents of the remaining accessions were moderate: higher and lower than those of Satsuma mandarin and Shiikuwasha, respectively.

PMF have well-known functions and bioactivities, including therapeutic effects against lifestyle-related diseases, such as anti-cancer, anti-tumor, and antihyperglycemic (Kawai et al., 1999; Lee et al., 2010; Minagawa et al., 2001; Miyata et al., 2008). In addition, they were reported to show effects against Alzheimer's disease (Nady et al., 2017; Onozuka et al., 2008). Hence, fruit with a high PMF content is considered to be good for the health and highly marketable. However, it is difficult to consume sufficient PMF by eating unprocessed fruits since the content in juice is very low (Nogata et al., 2006; Yamamoto et al., 2008). It is essential to develop processed fruit products with a large quantity of PMF derived from the peel.

Citrus fruit is also an important source of ascorbic acid (vitamin C), which is known as a functional component. In the present study, we used RQflex to measure the ascorbic acid content of the juice. This method is a very simple way to analyze the content. The values of the present study were almost the same as those of a previous study using the hydrazine method, a standard method to assay ascorbic acid, in the same accessions (Yamamoto et al., 2009). Data of the present study are considered to be reliable. The content of Satsuma mandarin was moderate (Yamamoto et al., 2009), and several accessions showed contents similar to that of Satsuma mandarin. Among the accessions used as materials, the contents of Shiikuwasha (10 and 11), Kabuchii (18, 19, 20, 21, and 22), and Shiikuu (30, 31, and 32) were very low. In contrast, 'Tarumizu 1gou' (7) and 'Fusu' (29) fruit showed a high ascorbic acid content. They are a good source of vitamin C.

The fruit of some accessions cultivated and grown in Kagoshima contained a large quantity of PMF. Among these, Ponkan and Tankan are important fruit crops in Kagoshima since they adapt to environmental conditions there and their general fruit quality is high. In addition, their fruit contains high PMF. This information is useful for the expansion of their sales. On the other hand, although local citrus show small-scale production, they are important materials for 'Chiiki-okoshi', activation of the economy and culture of a district, in some areas, and several kinds of fruit-processed products made from the peel and/or flesh of local citrus accessions, such as Shiikuwasha, Kuroshimamikan, Keraji, Kabuchii, Fusu, and Shiiku, have been developed. However, general fruit traits of the local accessions were inferior to those of the major cultivars. In general, they were found to have small fruit, a low percentage of flesh, and a low Brix value, although some possessed a distinct aroma (Hamada et al., 2017; Teramoto et al., 2017). Providing information on the health-promoting components of fruits is essential to add value to the products. The results of the present study provide useful information for promoting "Chiikiokoshi" in Kagoshima.

\section{Literature Cited}

Green, C. O., A. O. Wheatley, A. U. Osagie, E. Y. S. A. Morrison 
and H. N. Asemota. 2007. Determination of polymethoxylated flavones in peels selected Jamaican and Mexican citrus (Citrus spp.) cultivars by high-performance liquid chromatography. Biomed. Chromatogr. 21: 48-54.

Hamada, T., M. Hayasaki, H. Kitahara, K. Yamashita, A. Kariyazaki, F. Tani, S. Onitsuka and H. Okamura. 2017. Essential oil composition of citrus peels in Kikai-jima Island, Japan. Am. J. Essen. Oils Nat. Prod. 5: 12-15.

Inafuku-Teramoto, S., R. Suwa, Y. Fukuzawa and Y. Kawamitsu. 2011. Polymethoxyflavones, synephrine and volatile constitution of peels of citrus fruit grown in Okinawa. J. Japan. Soc. Hort. Sci. 80: 214-224.

Inafuku-Teramoto, S., M. Yamamoto, H. Kinjyo, A. Kitajima, K. Wada and Y. Kawamitsu. 2010. Local citrus genetic resources and their polymethoxyflavones content in northern part of Okinawa Island. Hort. Res. (Japan) 9: 263-271 (In Japanese with English abstract).

Kawai, S., Y. Tomono, E. Katase, K. Ogawa and M. Yano. 1999. Antiproliferative activity of flavonoids on several cancer cell lines. Biosci. Biotech. Biochem. 63: 896-899.

Lee, Y. S., B. Y. Cha, K. Saito, H. Yamakawa, S. S. Choi, K. Yamaguchi, T. Yonezawa, T. Teruya, K. Nagai and J. T. Woo. 2010. Nobiletin improves hyperglycemia and insulin resistance in obese diabetic ob/ob mice. Biochem. Pharma. 79: 1674-1683.

Minagawa, A., Y. Otani, T. Kubota, N. Wada, T. Furukawa, K. Kumai, K. Kameyama, Y. Okada, M. Fujii, M. Yano, T. Sato, A. Ito and M. Kitajima. 2001. The citrus flavonoid, nobiletin, inhibits peritoneal dissemination of human gastric carcinoma in SCID mice. Japan. J. Cancer Res. 92: 13221328.

Miyata, Y., T. Sato, K. Imada, A. Dobashi, M. Yano and A. Ito. 2008. A citrus polymethoxyflavonoid, nobiletin, is a novel MEK inhibitor that exhibits antitumor metastasis in human fibrosarcoma HT-1080 cells. Biochem. Biophysic. Res. Com. 366: 168-173.

Nady, B., B. Sahar, H. Solomon, A. Touqeer, D. Maria, N. M. Seyed, S. Eduardo and F. N. Seyed. 2017. Neuroprotective effects of citrus fruit-derived flavonoids, nobiletin and tangeretin in alzheimer's and parkinson's disease. CNS Neurolog. Disorder.-Drug Targets 16: 387-397.

Nogata, Y., K. Sakamoto, H. Shiratsuchi, T. Ishii, M. Yano and H. Ohta. 2006. Flavonoid composition of fruit tissues of citrus species. Biosci. Biotechnol. Biochem. 70: 178-192.

Onozuka, H., A. Nakajima, K. Matsuzaki, R. W. Shin, K. Ogino, D. Saigusa, N. Tetsu, A. Yokosuka, Y. Sashida, Y. Mimaki,
T. Yamakuni and Y. Ohizumi. 2008. Nobiletin, a citrus flavonoid, improves memory impairment and $\mathrm{A} \beta$ pathology in a transgenic mouse model of Alzheimer's disease. J. Pharmacol. Exp. Ther. 326: 739-744.

Park, G. L., S. M. Avery, J. L. Byers and D. B. Nelson. 1983. Identification of bioflavonoids from citrus. Food Tech. 37: 98-105.

Teramoto, S., T. Ninomiya and M. Yamamoto. 2017. The Bergamot aroma of local citrus 'Shiikuu' (Citrus sp.) originating from Kikai Island of Kagoshima Prefecture, Japan: Analysis of its essential oil characteristics and genetic background. Hort. Res. (Japan) 16: 239-248 (In Japanese with English abstract).

Yamamoto, M. and S. Tominaga. 2003. High chromosomal variability of mandarin (Citrus spp.) revealed by CMA banding. Euphytica 129: 267-274.

Yamamoto, M., M. Fukuda, T. Koga, T. Kubo and S. Tominaga. 2010. Examination of the origin of Keraji (Citrus keraji), local citrus of Kikaijima Island, Kagoshima Prefecture. Hort. Res. (Japan) 9: 7-12 (In Japanese with English abstract).

Yamamoto, M., T. Koga, M. Fukuda, T. Kubo and S. Tominaga. 2009. Ascorbic acid content and antioxidant activity of juice in various citrus accessions. Hort. Res. (Japan) 8: 273-279 (In Japanese with English abstract).

Yamamoto, M., R. Kouno, T. Nakagawa, T. Usui, T. Kubo and S. Tominaga. 2011. Isozyme and DNA analyses of local citrus germplasm on Amami Islands, Japan. J. Japan. Soc. Hort. Sci. 80: 268-275.

Yamamoto, M., R. Matsumoto, Y. Uechi, T. Ijichi, T. Kubo and S. Tominaga. 2008. The polymethoxy flavones content of local citrus accessions on the island of Kikaijima in Kagoshima Prefecture, Japan. Bull. Fac. Agr. Kagoshima Univ. 58: 1-7 (In Japanese with English abstract).

Yamamoto, M., K. Matsushima, T. Ijichi, Y. Uechi, S. Kawaguchi, H. Nakano, T. Nomura, O. Tanimura, T. Kubo and S. Tominaga. 2006. Exploration and preservation of local citrus germplasms in the Amami archipelago. Bull. Exp. Farm. Fac. Agr. Kagoshima Univ. 29: 5-11 (In Japanese with English abstract).

Yamamoto, M., A. Takakura, A. Tanabe, S. Teramoto and M. Kita. 2017. Diversity of Citrus depressa Hayata (Shiikuwasha) revealed by DNA analysis. Genet. Resour. Crop Evol. 64: 805-814.

Yoshioka, T., J. Higa, M. Arazaki and R. Matsumoto. 2001. Polymethoxy flavones content of peel and juice in Shiikuwashas. Mem. Japan. Soc. Hort. Sci. Kyushu Br. 9: 5 (In Japanese). 